\title{
Incidencia y prevalencia de la urolitiasis en España: Revisión de los datos originales disponibles hasta la actualidad
}

\author{
Sánchez-Martín FM, Millán Rodríguez F, Esquena Fernández S, Segarra Tomás J, \\ Rousaud Barón F, Martínez-Rodríguez R, Villavicencio Mavrich H.
}

\author{
Servicio de Urología. Fundación Puigvert. Barcelona.
}

Actas Urol Esp. 2007;31(5):511-520

\section{RESUMEN}

\section{INCIDENCIA Y PREVALENCIA DE LA UROLITIASIS EN ESPAÑA: REVISIÓN DE LOS DATOS ORIGINALES DISPONIBLES HASTA LA ACTUALIDAD}

Introducción: Los estudios de San Antonio y Somacarrera en 1977, y de Rousaud-Inmark en 1984, establecen las primeras cifras de incidencia y prevalencia de la urolitiasis en España, seguidas de otras apreciaciones epidemiológicas regionales o nacionales realizadas por otros autores.

Material y métodos: Se han seleccionado los trabajos con datos originales sobre incidencia o prevalencia, de ellos 5 son incidencia, 8 prevalencia y 3 contienen ambos. Diez grupos se basan en estudios poblacionales (San Antonio, Martín, Pedrajas, Rousaud-Inmark, Torres, Ripa, Romero, Grases, Alapont, Aíbar), 5 en estimaciones subjetivas (Solé-Balcells, Cifuentes, Puigvert, Serrallach, Conte) y uno es mixto (Somacarrera). Siete trabajos son de ámbito nacional y 9 son sobre zonas concretas. Se presenta un mapa con los enclaves de los diferentes estudios. Se ha realizado un registro de datos para proceder al cálculo de la media nacional de incidencia y prevalencia.

Resultados: La media de incidencia de la urolitiasis en España es del 0,73\%, correspondiente a 325.079 nuevos casos por año; y la de prevalencia es del 5,06\%, correspondiente a 2.233.214 casos totales.

Discusión: Los estudios más válidos desde el punto de vista metodológico se han basado en las encuestas a la población general. Un sesgo común en la mayoría de estudios es extraer el dato epidemiológico de los registros asistenciales retrospectivos. Aunque la mayoría de trabajos reflejan dificultades metodológicas, sus datos coinciden con los datos publicados internacionalmente, y muestran el interés por la epidemiología en el ámbito de la urolitiasis.

Palabras clave: Urolitiasis. Litiasis. Epidemiologia. Incidencia. Prevalencia.

\section{ABSTRACT}

INCIDENCE AND PREVALENCE OF PUBLISHED STUDIES ABOUT UROLITHIASIS IN SPAIN. A REVIEW

Introduction: San Antonio, Somacarrera (1977) and Rousaud-Inmark (1984) studies established the first data of incidence and prevalence about urolithiasis in Spain. Other regional or national epidemiologic details were given for several authors from 1977 to 2002.

Material and Method: Sixteen papers with original data about incidence or prevalence have been selected, 5 of them are about incidence, 8 about prevalence and 3 include both. Ten papers are based on poblational research (San Antonio, Martín, Pedrajas, Rousaud-Inmark, Torres, Ripa, Romero, Grases, Alapont, Aíbar), 5 on subjective estimations (Solé-Balcells, Cifuentes, Puigvert, Serrallach, Conte) and one include both (Somacarrera). Seven papers are nationwide and 9 are about local areas. A map with different location studies is presented and a data register show incidence and prevalence medians.

Results: The Spanish median urolithiasis incidence is 0'73\%, corresponding to 325,079 new cases per year; and the prevalence is $5^{\prime} 06 \%$, corresponding to $2,233,214$ cases.

Discussion: Methodologically the best epidemiologic studies about lithiasis are based on general population survey. The commonest slant is extract data from retrospective clinical registers. Most studies have significant methodological difficulties, but they reflected interest about epidemiology of stone disease in Spain. There is a concordance between Spanish results and international published data.

Keywords: Lithiasis. Urinary stone. Epidemiology. Incidence. Prevalence. 
$\mathrm{L}^{\mathrm{a}}$ a epidemiología es uno de los aspectos más interesantes de la enfermedad litiásica urológica. La dificultad para realizar estudios epidemiológicos sobre urolitiasis radica en la falta de registros sanitarios oficiales y en la propia naturaleza de la enfermedad, con un buen número de casos puntuales y sin recidiva. Las encuestas han servido como base para realizar estudios poblacionales, pero son los registros hospitalarios la fuente más empleada para la obtención de datos sobre frecuencia de la litiasis, reflejando únicamente los casos que han requerido atención hospitalaria. Sólo de forma excepcional se han realizado muestreos sobre todo el territorio español, siendo más común el estudio de áreas geográficas más concretas. La recogida y análisis de los datos de incidencia y prevalencia de la urolitiasis en España permite realizar un ejercicio matemático de aproximación al perfil epidemiológico de esta enfermedad.

El objetivo de este estudio es, en primer lugar, calcular una media de incidencia y prevalencia de la urolitiasis para todo el territorio nacional español, obtenido de la media de los datos publicados en los últimos 30 años y, en segundo lugar, establecer un mapa epidemiológico nacional de la urolitiasis con las regiones estudiadas.

\section{MATERIAL Y MÉTODOS}

La búsqueda de publicaciones de contenido epidemiológico en el ámbito geográfico español se ha realizado a través de la base de datos Pubmed (MEDLINE) y de las referencias bibliográficas que figuran en los propios artículos. Asimismo se ha realizado una búsqueda simple con el motor google.com con objeto de recoger trabajos divulgativos ausentes en las bases de datos médicas. La búsqueda ha correspondido al cruce de los términos "epidemiología” (y sus derivados, por ejemplo "epidemiológico") con "litiasis” y sus afines -tanto en español como en inglés- (por ejemplo "urolithiasis", "stone"), que estuvieran referidos a España. Una vez recopilados las referencias se han obtenido copias completas de las publicaciones. Se han excluido de la búsqueda las tesis doctorales, habida cuenta de la dificultad en consultar los originales, si bien se han recopilado algunos de sus datos para enriquecer la discusión.
Debido a que los trabajos confunden frecuentemente el concepto de incidencia y prevalencia, los datos se han agrupado en una $\mathrm{u}$ otra categoría una vez corregido su sentido. Con objeto de homogeneizar los datos en una u otra categoría, se ha considerado como incidencia el porcentaje de casos nuevos en el último año, y como prevalencia el porcentaje total de casos presentes en la población general en un momento dado. Se han convertido todos los datos a \% para homogenizar la revisión. A partir de los 16 trabajos con datos epidemiológicos originales se extrae la media aritmética de incidencia y prevalencia, que puede dar una idea aproximada de la frecuencia de la urolitiasis en nuestro país. Sólo se ha excluido un dato de prevalencia del 80-100\%, considerado outlier. La media se ha obtenido del sumatorio de todos los valores de incidencia y prevalencia locales o nacionales obtenidos. Se ha tomado como marco temporal del dato, el año señalado por el autor. Cuando el trabajo original se refiere a varios años, se ha tomado la moda del intervalo de años para asignar un año al trabajo concreto. En los trabajos donde no consta la fecha de la serie se ha tomado como referencia el año de su publicación. No ha sido posible realizar el cálculo con el censo del año correspondiente a cada una de las publicaciones debido a la falta de estimaciones censales anuales en la bases de datos del INE (sólo figuran estimaciones decenales entre 1900 y 1985). Debido a ello y, dado que los trabajos comprenden un amplio intervalo (de 1977 a 2004), los cálculos de incidencia y prevalencia general de la población española se han centrado en el año 1990, año central del intervalo 1977 a 2004. La población de derecho para 1990, obtenida del INE es de 39.887.140 habitantes, cifra en la que se ha basado el cálculo de habitantes litiásicos y año (incidencia) y total de habitantes afectados en un momento dado (prevalencia). No ha sido posible obtener del INE los datos sobre población de hecho para 1990.

\section{RESULTADOS}

A partir de 60 referencias obtenidas en el primer muestreo de búsqueda, se han seleccionado 45 trabajos que aportan datos epidemiológicos de interés, de los que 16 comunican datos originales 
incidencia y/o prevalencia. Se han incluido tanto los datos obtenidos a partir de estudios poblacionales como los basados en la opinión subjetiva del autor. Se entiende que son originales los datos fruto de la investigación $\mathrm{u}$ opinión propia de los autores. Existen otros 11 trabajos que citan datos ya publicados previamente, motivo por el que no han sido incluidos en el cómputo. De los 16 trabajos, 5 aportan datos originales de incidencia, 8 de prevalencia y, en 3 trabajos, se ofrecen ambos datos. Se obtiene un total de 19 datos $(8$ de incidencia +11 de prevalencia). Diez autores basan sus datos en estudios poblacionales (San Antonio ${ }^{1}$, Martín-Vivaldi ${ }^{3}$, Pedrajas ${ }^{6}$, Rousaud-Inmark ${ }^{7}$, Torres $^{8}$, Ripa ${ }^{11}$, Romero ${ }^{12}$, Grases ${ }^{13}$, Alapont ${ }^{15}$, Aíbar ${ }^{16}$ ), 5 realizan estimaciones subjetivas (SoléBalcells ${ }^{4}$, Cifuentes ${ }^{5}$, Puigvert $^{9}$, Serrallach ${ }^{10}$, Conte $^{14}$ ) y uno (Somacarrera ${ }^{2}$ ) es mixto (obtiene la incidencia a partir de un estudio poblacional, mientras el dato de prevalencia es subjetivo). Siete trabajos toman como universo la población general española (Somacarrera, Solé-Balcells, Cifuentes, Conte, Rousaud-Inmark, Puigvert y Serrallach), y los 9 restantes se refieren a zonas concretas (Fig. 1). Los trabajos se han publicado en revistas como Actas Urol Esp (6), Arch Esp Urol (2), JANO (1) y Medicine (1); y en monografia (2), libro de resúmenes 2 o capítulo de libro (1). En la Tabla 1 figuran los autores de los trabajos originales y el ámbito geográfico de su estudio, con el dato de incidencia y/o prevalencia.

La incidencia de urolitiasis en España, resultante del cálculo de la media sobre los datos previamente publicados, es del $0,73 \%$, es decir 737 casos cada 100.000 habitantes/año, que resultan 293.968 nuevos casos/año (para 1990, año

Tabla 1

Autores que han aportado datos originales sobre incidencia o prevalencia de urolitiasis en España. Computo de la media nacional de incidencia y prevalencia

\begin{tabular}{|c|c|c|c|c|}
\hline Autor & $\begin{array}{c}\text { Ámbito } \\
\text { geográfico }\end{array}$ & Año & $\begin{array}{c}\text { Incidencia } \\
\%\end{array}$ & $\begin{array}{c}\text { Prevalencia } \\
\%\end{array}$ \\
\hline San Antonio ${ }^{1}$ & Madrid & 1977 & - & 10,9 \\
\hline Somacarrera $^{2}$ & España & 1977 & 0,81 & 0,10 \\
\hline Martín-Vivaldi ${ }^{3}$ & Granada & 1979 & 2,95 & - \\
\hline Solé-Balcells ${ }^{4}$ & España & 1979 & -- & 3 \\
\hline Cifuentes ${ }^{5}$ & España & 1980 & 0,50 & - \\
\hline Pedrajas $^{6}$ & Granada & 1982 & 0,24 & 2'90 \\
\hline Rousaud-Inmark $^{7}$ & España & 1984 & $0,{ }^{\prime} 27$ & 4,16 \\
\hline Torres $^{8}$ & Granada & 1984 & - & $3, ’ 01$ \\
\hline Puigvert $^{9}$ & España & 1987 & -- & 2 \\
\hline Serrallach ${ }^{10}$ & España & 1987 & & $(80)$ \\
\hline Ripa $^{11}$ & Tudela & 1990 & 0,51 & - \\
\hline Romero $^{12}$ & Marina A. & 1990 & 0,28 & - \\
\hline Grases $^{13}$ & Baleares & 1990 & - & 14,3 \\
\hline Conte $^{14}$ & España & 1991 & -- & 10 \\
\hline Alapont ${ }^{15}$ & Hellín & 1996 & - & 0,26 \\
\hline Aibar $^{16}$ & Zaragoza & 2002 & 0,35 & -- \\
\hline CÓMPUTO TOTAL & & $\begin{array}{c}\text { AÑo } \\
\text { PROMEDIO }\end{array}$ & $\begin{array}{l}\text { INCIDENCIA } \\
\text { MEDIA }\end{array}$ & $\begin{array}{c}\text { PREVALENCIA } \\
\text { MEDIA }\end{array}$ \\
\hline \multirow[t]{2}{*}{ Habitantes España 1990: 39.887.140 } & & 1990 & $\begin{array}{c}0,73 \% \\
7,375 \% \text { o } \\
737 / 100.000\end{array}$ & $\begin{array}{c}5,063 \% \\
50,63 \% \text { o } \\
5.063 / 100.000\end{array}$ \\
\hline & & & $\begin{array}{c}293.968 \\
\text { casos/año }\end{array}$ & $\begin{array}{l}2.019 .485 \\
\text { total casos }\end{array}$ \\
\hline
\end{tabular}




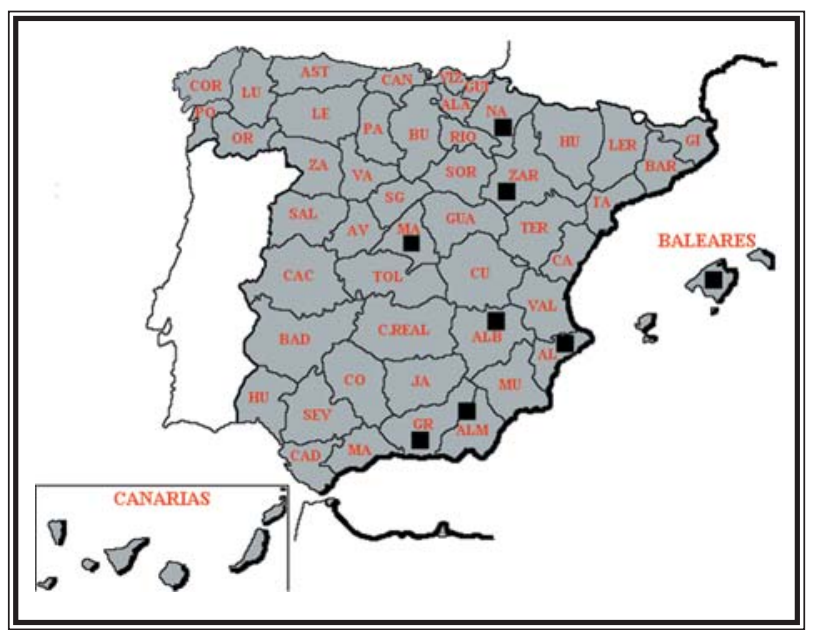

FIGURA 1. Provincias españolas que han sido estudiadas desde el punto de vista de la incidencia y prevalencia de la urolitiasis.

central del intervalo). Si se aplica la misma razón a la población de 44.108.530 habitantes correspondiente al año 2005 resultan 325.079 casos/año.

La prevalencia de urolitiasis en España, considerando los mismos criterios, es del $5,06 \%$, es decir 5.063 casos cada 100.000 habitantes, que resultan 2.019.485 personas afectadas entre la población total (total de casos para 1990, año central del intervalo). Extrapolándolo a la población de 2005 resultan 2.233.214 personas litiásicas entre el total de la población española (total de casos).

\section{DISCUSIÓN}

\section{Distribución geográfica}

Los estudios epidemiológicos sobre urolitasis en España parten de las primeras apreciaciones realizadas por San Antonio y Somacarrera ${ }^{2}$ en 1977 donde establecen una incidencia de 0,81\% casos/año. En 1984, la empresa Inmark, bajo la supervisión de A. Rousaud ${ }^{7}$, realiza el único estudio epidemiológico de ámbito nacional existente hasta la actualidad, estableciendo una incidencia $0,27 \%$ casos/año y una prevalencia de $4,16 \%$, cifras de referencia para revisiones posteriores. Desde entonces han ido apareciendo trabajos referidos a diferentes zonas de la geografia española, que incluyen 8 provincias ( 9 trabajos): Albacete, Alicante, Almería, Granada, Madrid, Navarra y Zaragoza. Los estudios son de ámbito comarcal (Hellin ${ }^{15}$, Marina Alta ${ }^{12}$, Tudela ${ }^{11}$ ), pro- vincial (Granada y comarcas de Almería ${ }^{3,6,8}$, Madrid $^{1}$, Zaragoza ${ }^{16}$ ) y de comunidad autónoma (Balear $\left.{ }^{13}\right)$, con los que puede establecerse un mapa con las zonas estudiadas hasta la actualidad (Fig. 1). Se trata de un número muy limitado de regiones valoradas, que pone de manifiesto la ausencia de estudios epidemiológicos suficientes que reflejen la realidad de la urolitiasis en nuestro país, tal como señalan diversos auto$\operatorname{res}^{17-19}$.

La zona geográfica más estudiada es la provincia de Granada ${ }^{3,6,8}$, tanto en zonas urbanas como en comarcas, representando 3 de los 16 trabajos. Pedrajas, Del Río y Torres (éstos con sendas publicaciones sobre frecuencia de urolitiasis en población gitana y no gitana) incluyen zonas rurales de la provincia de Almería lindantes con la de Granada ${ }^{6,20,21}$. Tanto los estudios de ámbito provincial (Madrid, Zaragoza) como los comarcales (Hellín, Marina Alta, Tudela) se basan en los registros propios del área de cobertura del hospital de referencia. Es común que las cifras de prevalencia o incidencia se basen en el número de pacientes asistidos por litiasis entre el total de la población o entre el número total de urgencias generales, lo que tiende a infravalorar las cifras reales ${ }^{17}$. Algunos estudios se limitan al estudio cristalográfico, extrapolando de forma inadecuada la incidencia de la enfermedad ${ }^{22}$.

El trabajo de Rousaud-Inmark se basó en un muestreo realizado en 15 provincias elegidas al azar (Santander, Pontevedra, Guipúzcoa, Zamora, Zaragoza, Teruel, Barcelona, Madrid, Cáceres, Toledo, Albacete, Alicante, Sevilla, Granada y Gran Canaria), incluyendo 67 municipios estratificados por número de habitantes. Cuatro de ellos eran grandes capitales, 13 municipios de entre 100.000 y 500.000 habitantes, 10 entre 30.000 y $100.000,15$ entre 5000 y 30.000 , y 25 entre 100 y 5.000 . No se dispone de los datos de cada uno de los municipios, pero se analizó la distribución de la enfermedad según número de habitantes, hallándose que la mayor prevalencia de la enfermedad se registra en las localidades más pequeñas $(5,21 \%)$ y más grandes $(5,12 \%)$ mientras que las intermedias son menos propensas, especialmente las comprendidas entre 100.000 y 500.000 habitantes $(2,87 \%)$. 


\section{Incidencia y prevalencia}

Las consultas on line a la web del INE no aportan datos concretos sobre incidencia o prevalencia de la urolitiasis en España, carencia que se enmarca dentro del escaso desarrollo de la epidemiología en nuestro país. Existen datos internacionales de prevalencia que, excepto Italia $^{23} \mathrm{y}$ USA $^{24}$ (por encima del 10\%), se sitúan en rangos similares a los del estudio de Rousaud-Inmark. La media nacional del 5,06\% obtenida en el presente trabajo, está en los rangos de las diversas publicaciones internacionales (Tabla 2). Respecto a la incidencia, la media nacional de 0,73\%, está cercana a los datos de aquellos autores que han realizado estudios de campo, como Somacarrera, Ripa, Romero, Aíbar (entre 0,8 y 0,3\%), o a los estudiados sobre USA (incidencia hospitalaria: $0,16-0,28 \%$ y año $)^{25}$ y Países Nórdicos $(0,05 \%$ $0,2 \%$ y año) ${ }^{26,27}$, si bien la incidencia es un dato poco reflejado en la literatura. A pesar de las limitaciones de la metodología del presente trabajo es sorprendente la verosimilitud de las cifras medias nacionales obtenidas.

\section{Tabla 2}

Estudios internacionales de prevalencia de la urolitiasis (tomado de Ibarz)

\begin{tabular}{lcc}
\hline País & Año & $\begin{array}{c}\text { Prevalencia } \\
\text { \% }\end{array}$ \\
\hline España & 1980 & $5,06^{*}$ \\
Gran Bretaña & 1977 & 3,4 \\
USA & 1978 & 12 \\
Holanda & 1978 & 4,4 \\
Hungría & 1979 & 1,2 \\
Italia & 1979 & 13 \\
Suecia & 1980 & 5 \\
Alemania & 1982 & 4 \\
Austria & 1986 & 4,8 \\
Francia & 1989 & 5,6 \\
\hline
\end{tabular}

*Media nacional obtenida por cómputo de datos disponibles entre 1977-2002.

En la ponencia del XLII Congreso Nacional de Urologia de 1977 (Santander), Somacarrera et al. ofrecieron un dato subjetivo de prevalencia del uno por mil $(0,1 \%)$, que puede "aumentar con la variación en los climas”. Respecto a la incidencia aportan un dato del $0,81 \%$, obtenido de 1.226 ingresos por litiasis durante 1975 , sobre el total de 150.022 ingresos generales, para una población atendida de 4.511.786 habitantes ${ }^{2}$. El dato es equívoco en cuanto se calcula la incidencia, no en base a la población general, sino al número de ingresos totales. Calculado sobre la población general la incidencia correcta en este trabajo hubiera correspondido con más propiedad al 0,027\%. Los autores afirman además que sólo es necesario ingresar uno de cada 5 casos de litiasis, por lo que es plausible que la incidencia pudiera asimismo multiplicarse por 5. En la zona de influencia del Hospital Clínico de Madrid la frecuencia (se interpreta prevalencia) queda ajustada al 15,13\%, resultante de 793 enfermos litiásicos sobre 5.241 del total de ingresos urológicos, en el lapso $1972-76^{2}$. En la misma ponencia San Antonio et al. aportan una cifra de "incidencia general" o prevalencia del 10,9\%, basada en 554 enfermos litiásicos entre un total de 5.038 enfermos urológicos atendidos entre 1970-76 en el mismo centro. Además establecen un mapa de frecuencia de litiasis en base a los casos que, desde las diferentes provincias españolas, son derivados a su hospital. Los autores advierten que los datos "no son reales en cuanto a demostrar la verdadera distribución geográfica” pero realizan una aproximación epidemiológica de ámbito nacional de la urolitiasis. Los datos, aceptando los errores de cálculo y los sesgos, son el primer intento de cuantificar con carácter objetivo la epidemiología de la litiasis en España ${ }^{1}$.

Torres, del grupo del Hospital Virgen de las Nieves de Granada, realiza en el año 1984 el estudio poblacional local más profundo que ha tenido lugar en España. Se realizaron 16.492 entrevistas puerta a puerta, extrayendo un importante banco de datos que se tradujo en diversas publicaciones nacionales e internacionales de éste ${ }^{8,21}$ y otros autores del grupo ${ }^{6,20}$. En su publicación del Journal of Urology, el autor reconoce las limitaciones del valor epidemiológico de su estudio ${ }^{21}$. El mérito del trabajo es destacado en cuanto representa un estudio pormenorizado de la frecuencia de urolitiasis en la población general de su provincia, incluyendo factores como edad, género, grupo racial, alimentación, climatolología o composición del agua. Diferencia los conceptos prevalencia e incidencia, pero no utiliza estas denominaciones, 
sino "incidencia de historia de litiasis" para incluir aquí los sujetos con antecedentes recientes o pasados de litiasis, lo que resulta más cercano al concepto de prevalencia. Cifra erróneamente la "incidencia de historia de litiasis" en $3,01 \%^{28}$ ya que si se examinan otras publicaciones de este autor se aprecia que dicho porcentaje corresponde únicamente a los varones ${ }^{21}$. Contando hombres y mujeres, de cualquier edad y de cualquier raza la prevalencia, a partir de los propios datos del autor, corresponde al 2,75\%.

El trabajo de Martín-Vivaldi et al. se refiere a una prevalencia del $2,95 \%$, pero el dato corresponde a la pequeña población de Armillas, donde se encuestó a 2.147 familias, 9.575 individuos $^{3}$. Esta localidad fue usada como "grupo control" para comparar los datos de prevalencia del total de la población asignada al Hospital Virgen de las Nieves de Granada. De forma subjetiva los autores afirman que "una octava parte de la población padece algún episodio litiásico a lo largo de su vida". Pedrajas, en su tesis de 1983 y posterior publicación en Actas Urológicas Españolas se basa en esta misma población ${ }^{6}$, ya que se trata del mismo grupo de investigadores, estableciendo una cifra de "incidencia bianual" (1981-82) en la provincia de Granada del 0,24\%, dato obtenido a partir de las 1.929 litiasis demostradas con RX entre el total de pacientes atendidos en urgencias de los hospitales de referencia de Granada. Señala que la urgencias por "cólicos renales" se elevaron a 8.500 , lo que presupone cifras superiores de incidencia. Este autor colaboró en la supervisión del estudio Inmark y es el único urólogo español que ha realizado una tesis de contenido epidemiológico sobre litiasis.

Rousaud presenta en 1986 un estudio epidemiológico nacional sobre litiasis ${ }^{7}$ que ha sido el principal referente para revisiones posteriores. Promovido por el Grupo de Litiasis de la Asociación Española de Urología, el estudio fue realizado por la empresa CP Inmark SA de estudios y estrategias. Los datos se obtuvieron de un amplio muestreo nacional, empleando un sistema de encuesta domiciliaria a 2.238 personas (600 familias) durante el periodo comprendido entre abril de 1985 y febrero de 1986. El trabajo fue presentado en un acto público pero no llegó a plasmarse en ningún soporte perdurable desde el punto de vista bibliográfico. A pesar de la dificultad para consultar el dossier original, muchos de las publicaciones aparecidas posteriormente sobre esta temática citan los datos de Inmark como referentes, unas veces atribuyéndolos a Rousaud $^{14,19}$ y otras a la AEU ${ }^{12,17}$. Es evidente la importancia del estudio y el hecho de que los dossiers obran en poder de los diversos líderes de opinión que han ido reproduciendo los datos cuando han escrito sobre temática epidemiológica. El estudio reveló que "la urolitiasis afecta al $15,1 \%$ de los hogares españoles, y al 4,16\% de la población residente" en los mismos (prevalencia), lo que corresponde a 1.612.547 afectados entre el total de la población española a diciembre de 1985 (4,5\% de hombres frente a 3,82\% de mujeres). El método para determinar la prevalencia es fiable, pero no sucede lo mismo con el dato de incidencia, obtenido de la extrapolación de las cifras: En un anexo del dossier se afirma que se producen 180.000 a 200.000 casos nuevos al año. Aplicado a la población de hecho de 1981 (última disponible en el INE en ese periodo de tiempo) de 37.742.561 habitantes, representa un $0,49 \%$ y no el 0,27 que se ha ido transcribiendo en revisiones posteriores. Si se parte de la base de que un total de 1.612.547 personas tienen o han tenido litiasis y, de ellas el 43,9\% en los últimos 4 años, resulta un total de 707.908 en 4 años, es decir 176.977 casos en un año, cifra correspondiente a la incidencia. Estos 176.977 nuevos casos, para el censo estimado en 1985 de 38.588.296 habitantes (según un cálculo de proyección de población, desde la media de censos entre 1981 y 1990), arrojan una incidencia del $0,45 \%$, cifra que tampoco concuerda. Si, como afirma Arrabal en diversas publicaciones ${ }^{30,31}$ refiriéndose al estudio de Rousaud-Inmark, se toman 107.602 pacientes nuevos al año sobre la población estimada para 1985, resulta efectivamente el dato de $0,27 \%$, que es el que viene reflejándose en las sucesivas revisiones, si bien dicho número de pacientes no figura en el dossier original. En cualquier caso se trata de las cifras de referencia utilizadas habitualmente en revisiones sobre litiasis de autores españoles ${ }^{16,29,32,33}$. No obstante es recomendable que, para revisiones futuras que se refieran al estudio RousaudInmark, se tenga en cuenta la cifra de incidencia 
del 0,45\%. En otra publicación el mismo Rousaud cita su propio trabajo y redondea diciendo que "más de 1.700 .000 españoles han sufrido o están sufriendo de cálculos urinarios", añadiendo que "el próximo año 103.727 españoles se sumarán" a esta cifra ${ }^{34}$. Si consideramos que se refiere al año 1993 (población de derecho de 39.790.955 habitantes), resulta una incidencia del $0,26 \%$, más cercana al dato clásico del $0,27 \%$.

Para Conte et al., cerca de un $10 \%$ de la población padece o ha padecido de piedras en el riñón y, en Baleares, se supera ese porcentaje, llegando hasta el $14 \%$ de la población ${ }^{14}$. La referencia al 10\%, como cifra de prevalencia es comentada de forma habitual, quizás a partir de los datos clásicos de Boyce ${ }^{35}$ y Mandel ${ }^{24}$. El dato de prevalencia para Baleares coincide con el del trabajo de Grases et al. en que se establece una prevalecía autonómica del $14,3 \%{ }^{13}$, muy superior a las cifras de los demás autores (Tablas 1 y 2). Sólo San Antonio et al., en 1977, establecen una prevalencia del 10,9\%1. El estudio balear consistió en una encuesta a la población general, que completaron 847 encuestados entre juniodiciembre de 1990, matizando variaciones con la edad: para 18-30 de 4,5\%, para 31-50 años de $14,9 \%$ y para mayores de 50 años de $19,4 \%$. Desde el punto de vista metodológico este es uno de los trabajos mejor diseñados y con resultados más fiables. También el trabajo de San Antonio fue basado en una encuesta.

Los datos subjetivos son comunes en los líderes de opinión. Solé-Balcells fue el primer autor que empleó el término "prevalencia" en un estudio sobre epidemiología de la litiasis, aunque aportó un dato del $3 \%$, sin definir la fuente ${ }^{4}$. El trabajo, de un diseño estadístico impecable para la época (1979), se centra en la litiasis recidivante $\mathrm{y}$ establece consideraciones sobre la edad, composición y necesidad de cirugía. En 1980, Cifuentes afirma que si se admite solamente una proporción del $0,5 \%$ es posible que en nuestro país haya más de 180.000 personas a lo largo del año con urolitiasis" ${ }^{5}$. En 1987, Puigvert informa que "ha aumentado la frecuencia... de la calculosis renal en adultos que, según algunos, sobrepasa el $2 \%$ de la población", pero no aporta la fuente bibliográfica ${ }^{9}$. Ese mismo año Serrallach et al. señalan que "a lo largo de la vida de una persona, raramente deja de contabilizarse, por lo menos, un episodio de litiasis renal" y matiza: "de cada 10 personas que transitan por una calle de cualquier ciudad, 8 por lo menos han tenido litiasis urinaria y que, de éstos, sólo 2 se han traducido de forma clínica" ${ }^{10}$, lo que significa una prevalencia del $80-100 \%$, muy superior a la del resto de autores.

En estudios de ámbito local se han señalado cifras de frecuencia de la enfermedad basada sobre todo en los casos atendidos en los correspondientes hospitales, por ello suelen reflejar una cifra inferior a la real. El trabajo titulado "epidemiología de la litiasis urinaria" de Alapont et al. comprende sólo Hellín (Albacete) y su comarcalidad $^{15}$. Reúne una serie de datos de interés epidemiológico, analizando las visitas por litiasis a su hospital durante los años 1992-99, pero ofrece los datos en forma equívoca al calificarlos de "incidencia acumulada" para un año determinado (1998), que resulta ser del 0,26\%. Excluyendo los casos pediátricos (1-15 años) alcanza el $0,33 \%$. Por edades la curva de "incidencia" presenta un trazado bimodal, con picos alrededor de los 35 y 65 años. Después afirma textualmente una "prevalencia" por género de 0,39\% para varones y 0,26\% para mujeres. Los datos reflejan con más propiedad la prevalencia que la incidencia. Aíbar et al. hallan una incidencia de litiasis del 0,35\% en Zaragoza (área de salud III), cifra obtenida a partir de los cálculos recibidos para análisis cristalino en el laboratorio durante un año ${ }^{16}$. Existe el sesgo de incluir únicamente los casos que reciben análisis cristalográfico (a pesar de que también disponen de la cifra de "sucesos litiásicos"). Ripa et al. ofrecen una incidencia del $5,1 \%$ para la población del área de salud V de Navarra, correspondiente al Hospital de Tudela ${ }^{11}$. Calculan el dato a partir del número de pacientes atendidos por cólico renal en su hospital: 785 casos en dos años sobre 76.286 habitantes, lo que arroja una incidencia de 0,5\% habitantes/año. Al excluir los casos pediátricos y la incidencia sube al $6,42 \%$. Romero et al., aportan una frecuencia de litiasis del $0,28 \%$ para la comarca de la Marina Alta (Alicante), a partir de los casos de litiasis vistos durante un año en la consulta, lo que puede interpretarse como un 
dato de incidencia ${ }^{12}$. Como es habitual en estos trabajos aparece el sesgo de contabilizar únicamente los casos atendidos en el hospital. El concepto de incidencia queda asimismo sesgado en el trabajo de Romero al no haberse diferenciado los casos nuevos en un año, de los que debutaron antes.

Además de los 16 trabajos estudiados, existen otras 11 publicaciones en que los autores aportan una cifra de incidencia o prevalencia extraída de trabajos propios publicados previamente (Conte ${ }^{14}$, Torres ${ }^{21}$ ) o de estudios de otros autores de proximidad (del Río ${ }^{20}$ ) o de ámbito nacional, donde los datos más citados son los de RousaudInmark, al que se refieren en sus revisiones autores como Aibar ${ }^{16}$, Alcover ${ }^{29}$, Antúnez ${ }^{19}$, Arrabal ${ }^{31}$, Conte $^{33}$, Ibarz ${ }^{17}$, Lancina ${ }^{32}$ y Muñoz ${ }^{36}$. En el año 2000 Arrabal realizó un estudio sobre las LEOC realizadas en las diversas comunidades autónomas que, si bien no aporta cifras de prevalencia general sobre la enfermedad litiásica, arroja una idea de la prevalencia de la litiasis clínicamente activa (que suele recibir tratamiento con LEOC). Asumiendo el sesgo de quedar limitado a la litiasis tratada con LEOC se obtienen cifras de 1,09\% para Cataluña, 1,26\% para Madrid, 0,63\% para Valencia, 0,54\% para Galicia, 0,83\% para el País Vasco, 0,33\% para Castilla y León, 0,39 para Castilla La Mancha, 0,49\% para Canarias, 1,48\% para Aragón, 0,38 para Asturias, 0,43 para Extremadura, 0,31\% para Murcia, 0,40 para Baleares y $0,76 \%$ para Navarra ${ }^{30}$.

Los sesgos que afectan a las cifras en muchas de las publicaciones se derivan de la ausencia de registros epidemiológicos oficiales obtenidos a partir de la declaración de enfermedades (inexistentes en España para enfermedades como la urolitiasis), por lo que los datos epidemiológicos parten de los registros hospitalarios o de asistencia primaria, lo que refleja más los casos litiásicos atendidos que la frecuencia real de la enfermedad en la población general. La antigüedad de la mayoría de trabajos (todos excepto uno, anteriores a 1997) aporta asimismo el sesgo inherente a una realidad social diferente. La incidencia y prevalencia de litiasis ha aumentado en los últimos años ${ }^{37,38}$, por lo que puede suponerse una mayor frecuencia en la actualidad que la registrada en los estudios revisados. En el trabajo de
Yoshida et al. la incidencia anual de primer episodio litiásico para 1995 es del 0,068\% (68,9 por 100.000 habitantes) cifra superior a la de 0,054\% en 1965. La universalización de los conceptos de incidencia y prevalencia (inclusión del total de la población) implica asimismo una infravaloración de la misma. Por ejemplo, si se excluye la población de edad menor a 15 años (como ha hecho Alapont) la prevalencia sube al $0,33 \%{ }^{15}$. Según Andersen et al. la epidemiología de la enfermedad depende de factores intrínsecos y extrínsecos muy variables, por lo que la cifra definitiva es muy dependiente del momento y lugar en que se realice la medición ${ }^{39}$. Como señalan Ibarz ${ }^{17}$, Arrabal $^{31}$ y Antúnez ${ }^{19}$ en sus revisiones, las dificultades para lograr validez en estudios epidemiológicos son múltiples, habiendo una gran tendencia a obtener cifras que subestiman la realidad de la enfermedad. Otros sesgos pueden magnificar la cifra, como cuando se segmenta la prevalencia por etnias, siendo para la población gitana del $1,4 \%$, y para la no gitana del 3,01\% $8,20,21$. La prevalencia, calculada como "incidencia acumulada" (casos con litiasis actual sumados a aquellos que tienen antecedente de litiasis pero no litiasis actual) aporta asimismo un importante número de casos ya resueltos, con la consiguiente sobreestimación de la cifra total de casos.

\section{CONCLUSIONES}

De entre 45 publicaciones de contenido epidemiológico sobre urolitiasis en España se han publicado los 16 trabajos que aportan datos originales sobre incidencia o prevalencia. La media obtenida de todo ellos, arroja una incidencia del 0,73\% correspondiente a 325.079 nuevos casos por año; y una prevalencia de 5,06\%, correspondiente a 2.233.214 casos totales. Algunos de los trabajos reflejan únicamente una opinión subjetiva del autor, generalmente un líder de opinión, pero otros responden a estudios relativamente bien diseñados. Destacan los de Somacarrera y San Antonio (1977), Torres , Pedrajas y resto de autores granadinos (1977-84), Rousaud-Inmark (1984) y Conte y Grases (1994). Los estudios más válidos desde el punto de vista metodológico se han basado en las encuestas a la población general. Un sesgo común en la mayoría de estudios es extraer el dato epidemiológico a partir de las visitas por litiasis regis- 
tradas en el hospital. Aunque la práctica totalidad de los trabajos presenta las dificultades metodológicas, propias de este tipo de estudios, es reconocible el esfuerzo de los diversos autores para explorar sus ámbitos de competencia geográfica en busca de datos epidemiológicos sobre urolitiasis.

\section{Agradecimientos}

Gracias al Dr. A. Rousaud por facilitar amablemente el original del Estudio sobre Urolitiasis en España, elaborado por CP Inmark, S.A. en 1986.

\section{REFERENCIAS}

1. San Antonio AJ, Somacarrera E. Clínica de la litiasis renal. En Litiasis renal. Ponencia al XLII Congreso nacional de urología. Act Urol Esp. 1977; 2: 5-6..

2. Somacarrera E. Generalidades. En Litiasis renal. Ponencia al XLII Congreso nacional de urología. Santander. Actas Asoc Esp Urol. 1977;9(1):7-11.

3. Martín-Vivaldi J, Godoy J, Gamiza Berjon F, Cohen JS. estudio epidemiológico de la litiasis renal litiasis en Granada. XVIII Congres SIU. Paris. 1979 Kongresbericht. Tome 2. 46-47.

4. Sole-Balcells F, Pinto B, Ruiz-Marcellan FJ, Bernshtam J: Epidemiología de la litiasis renal recidivante. XVIII Congres SIU. Paris. 1979 Kongresbericht. Tome 2. 77-78.

5. Cifuentes L. Litiasis del aparato urinario. Medicine (Esp). 46: 2861. 1980.

6. Pedrajas Gamiz A, Arrabal Martín J, Miján Ortiz, J Rodríguez Rebollo T. Epidemiología de la urolitiasis en la provincia de Granada. Arch Esp Urol. 1984 Nov-Dec;37(6): 491-501.

7. CP inmark AS de estúdios y Estratégias. Estudio sobre la urolitiasis en España. Asociación española de Urología. Grupo de Urolitiasis. Promotor: Rousaud A. Supervisores: Rousaud A, Pedrajas A, Patrocinio: Centro de estudios Welcome España. CP Inmark SA de Estudios y Estrategias. Febrero. 1986

8. Torres RamirezC, del Rio Samper S, Zuluaga Gómez A, Espejo Maldonado E, Puebla Caverino M. Influencia del calcio de la dieta en la incidencia de litiasis renal. Arch Esp Urol. 1984 Mar-Apr;37(2):123-33.

9. Puigvert A: El mal de piedra del riñón.Salvat Ed. SA. Barcelona. 1987.

10. Serrallach N, Aguiló F, Rusconi A, Serrate RG, Torrecilla C. Litiasis urinaria. Tema monográfico. JANO. Vol XXXIII. 1824. 1987:838-840.

11. Ripa Saldias L, Delpon Pérez E, Romero Fernández FJ. Epidemiología de la litiasis urinaria en la Ribera de Navarra. Actas Urol Esp. 1995 Jun;19:459-66.

12. Romero Pérez P, Amat Cecilia M. Epidemiología de la urololitiasis en la region de la Marina Alta (Alicante). Actas Urol Esp. 1992;Jun;16(6):455-61.

13. Grases F, Conte A, March JG, Genestar C, Costa-Bauzá A Martin M, Vallescar R. Epidemiology of urinary stone disease in the Balearic Islands Community. Int Urol Nephrol. 1994;26(2): 145-50.

14. Conte A, Genestar C, Grases F, March JG, Martín M, vallescar R: Epidemiología de la litiasis renal en la Comunidad Balear. Consellería de Sanitat i Seguretat Social. Govern Balear. Palma de Mallorca. 1991. www.uib.es/servei/comunicacio/sc/projectes/arxiu/nousprojectes/litiasi/litiasicast.pdf
15. Alapont Pérez FM, Galvez Calderón J, Varea Herrero J, Colome Borros G, Olaso Oltra A, Sanchez Bisono JR. Epidemiología de la litiasis urinaria. Actas Urol Esp. 2001 May;25(5):341-349.

16. Aíbar MA, Gutierrez AP, Rodrigo MP, Laborda K, Hernández $\mathrm{AB}$, Blasco G.Litiasis renal en area III de Zaragoza: Bioquímica y epidemiología. Actas Urol Esp. 2004; 28(9):661-665.

17. Ibarz L, Ruiz Marcellan FJ: Aspectos históricos y epidemiología de la litiasis renal. Urol Integr Invest, 1997;2:363.

18. Usón AC, Romero F, Pedrajas A, Somacarrera E. Contribución al conocimiento epidemiológicos de la litiasis renoureteral en tres regiones de España. XVIII Congres SIU. Kongresbericht. Paris. 1979. Tome 2:45-46.

19. Antúnez F, Pedrajas A, Lancina JA. Epidemiología de la litiasis urinaria. En Criterios clínicos y tratamiento actual de la litiasis urinaria. Arrabal M, Lancina JA, García M Editores. Tema Monográfgicos al LV Congreso Nacional de Urología. Vigo. 1990.

20. Del Rio Samper S, Torres Ramirez C, Zuluaga Gómez A, Espejo Maldonado E, Puebla Ceverino M. Estudio epidemiológico comparativo de la litiasis renal en poblaciones gitanas y no gitanas: influencia de la dieta. Actas Urol Esp. 1984 Jul-Aug;8(4):265-278.

21. Torres Ramirez C, Fernandez Morales E, Zuluaga Gómez A, Galvez Alcaraz L, Del Rio Samper S. An epidemiological study of renal lithiasis in gypsies and others in Spain. J Urol. 1984 May;131(5):853-856

22. Mateos J, Avila S, García Cuerpo E, Berenguer A, Avila J, Lovaco F. Incidencia de la litiasis renal. Actas Urol Esp. 1980;4(1):7-10.

23. Pavone M, Miano L: Epidemiology of urolithiasis in Italy. XVIII Congrés de la Societé Internationale d'Urologie. París. 1979. Citado por Ibarz L, Ruiz Marcellán FJ, Aspectos históricos y edpidemiología de la litiasis renal. Urol Integr Invest. 1997;2.363.

24. Menon M, Resnik MI: Urinary lithiasis: Etiology, diagnosis and medical management. In Retick AB, Darracott E, Wein AJ (Eds.): Campbell's Urology. Saunders. Philadelphia. 2002. p3232. Citan a Mandel NS, Mandel GS: Urinary tract stone disease inte United status veteran population. I \& II. J Urol. 1989; 142:1513-1521.

25. Schey HM, Corbett WT, Resnick MI: Prevalence rate of stone disease in Forsyth County. North Carolina during 1977. J Urol. 1979; 122(3):288-291.

26. Juuti M, Heinonen OP.Incidence of urolithiasis leading to hospitalization in Finland. Acta Med Scand. 1979;206(5): 397-403.

27. Laerum E: Urolithiasis in general practice. Scan J Urol Nephrol. 1983. 17: 313-319.

28. Torres C, Del Rio S, Zuluaga A, Colloridi A, Puebla M. Epidemiología de la litiasis renal: influencia de la dieta. Arch Esp Urol. 1984 Jul-Aug;37(4):309-327.

29. Alcover J, Rousaud A, Ruiz Marcellan FJ, Serrallach N, Serrate R. Efectos adversos de las ondas de choque. Tema monográfico. LVII Congreso Nacional de Urología. 1992.

30. Arrabal Martín M. .Grupo de litiasis de la AEU.Litotricia extracorpórea en España en el siglo XX Actas Urol Esp. 2000;24(9) 699-708.

31. Arrabal M. Aspectos historicos, epidemiologicos y terapeuticos de la litiasis urinaria. Arch Esp Urol. 2001 Nov; 54(9): 845-50.

33. Lancina A. Coste, análisis del coste y coste-eficacia de la profilaxis médica en pacientes con urolitiasis. En Arrabal M, Conte A, Lancina A, Ozonas M, Rousaud A. Mesa Redonda. Aspectos económicos del tratamiento de la litiasis. Actas Urol Esp. 1995; 19: 486-513. 
33. Conte A. Aspectos económicos del tratamiento de la litiasis. En Arrabal M, Conte A, Lancina A, Ozonas M, Rousaud A. Mesa Redonda. Aspectos económicos del tratamiento de la litiasis. Actas Urol Esp. 1995; 19: 486-513.

34. Rousaud A: Prefacio. En Urolitiasis: metodología diagnóstica y terapéutica. Rousaud A y Barceló P 1992 Eds. Pulso ediciones. Barcelona.

35. Menon M, Resnik MI: Urinary lithiasis: Etiology, diagnosis and medical management. In Retick AB, Darracott E, Wein AJ (Eds.): Campbell's Urology. Saunders. Philadelphia. 2002. p3232. Citan a Boyce WH, Garvey FK, Starwcutter $\mathrm{HE}$ : incidente of urinary calculi among patients in general hospital: 1948-1952. Jama. 1965. 161: 1437-42.

36. Muñoz Martínez JA. Litiasis renal Oxalocálcica. Avances en la comprensión de su etiología y mejoras en la metodología analítica para su estudio. Tesis Doctoral. Universitat Autónoma de Barcelona. Departament de Química. Octbre. 2004.

37. Trinchieri A. Epidemiological trends in urolithiasis: impact on our health care systems. Urol Res. 2006 Apr;34(2):151156.
38. Daudon M. Epidemiology of nephrolithiasis in France. Ann Urol (Paris). 2005 Dec;39(6):209-231.

39. Drach GW. Urinary lithiasis: Etiology, diagnosis and medical management. In Walsh PC, Retick AB, Stamey TA, Darracott E (Eds.): Campbell's Urology. WB Saunders Co. Philadelphia. 1992. p 2086. Cita a Andersen DA: Environmental factor in the etiology of urolithiasis in urinary calculi. In Cifuentes L, Rapado A, and Hodgkinson A, (Eds.): Urinary Calculi: International Symposium on Renal Stone research. New York. S Karger 1973. p130.

Correspondencia autor: Dr. F.M. Sánchez Martín Servicio de Urología. Fundación Puigvert Cartagena, 340-350. 08025 Barcelona Tel.: 934169700

E-mail autor: fsanchez@fundacio-puigvert.es

Información artículo: Original - Litiasis

Trabajo recibido: febrero 2007

Trabajo aceptado: marzo 2007 